\title{
Auras and the risk of seizures with impaired consciousness following epilepsy surgery: implications for driving
}

\author{
Sam Fairclough, ${ }^{1}$ Aidan G O'Keeffe, ${ }^{2}$ Jane de Tisi, ${ }^{1}$ John S Duncan ${ }^{1,3}$
}

'Department of Clinical and Experimental Epilepsy, UCL Institute of Neurology, London, UK

${ }^{2}$ Department of Statistical Science, UCL, London, UK ${ }^{3}$ Chalfont Centre for Epilepsy, Chalfont St Peter, UK

Correspondence to Professor John S Duncan, Department of Clinical and Experimental Epilepsy, UCL Institute of Neurology/National Hospital for Neurology and Neurosurgery, London WC1N 3BG, UK; j.duncan@ucl.ac.uk

Received 2 June 2017 Revised 19 September 2017 Accepted 20 November 2017 Published Online First 9 December 2017
Check for updates

To cite: Fairclough $S$ O'Keeffe AG, de Tisi J, et al. J Neurol Neurosurg Psychiatry 2018;89:599-602.

\section{ABSTRACT}

Objective To calculate the chance of a seizure in the next year (COSY) for seizures with impaired awareness in those experiencing auras only, those with no seizures and those with continuing seizures. Epilepsy surgery is an effective treatment for refractory focal epilepsy. Driving is an important factor affecting quality of life. In the UK, driving is not permitted if focal seizures with no impairment of awareness (auras, simple partial seizures) continue, if there is a prior history of seizures with impaired awareness, as will invariably be the case in those having epilepsy surgery. Current UK driving regulations allow driving if COSY is less than $20 \%$.

Method We calculated COSY in 819 epilepsy surgery patients with up to 25 years follow-up. Each patient year was graded on the The International League against Epilepsy surgery outcome scale.

Results Patients who were entirely seizure-free for 1,2 and 3 years had COSY of $4.9 \%, 3.5 \%$ and $2.4 \%$ respectively. Patients with only auras within the last 1 , 2 or 3 years had a COSY of $11.3 \%, 9.2 \%$ and $7.8 \%$ respectively.

Conclusions Individuals with auras only after epilepsy surgery had a higher COSY than those who were seizure-free. If a COSY of below $20 \%$ is regarded as an acceptable risk, it may be suggested that those with auras only in a given year be allowed to drive. The relative risk of these patients causing accidents is lower than population groups such as those aged $<25$ or $>75$ years, who are permitted to drive.

\section{INTRODUCTION}

Neurosurgery is a successful treatment for medically intractable focal epilepsy. ${ }^{12}$ The International League against Epilepsy (ILAE) surgery outcome scale provides six categories of seizure frequency which can be applied annually. Crucially, a distinction is made between those who are entirely seizurefree and those who have auras (focal seizures with retained awareness, simple partial seizures). ${ }^{3}$

After epilepsy surgery, patients have a dynamic range of outcomes, including prolonged remissions, remissions and relapses. ${ }^{2}$ In this study, $10 \%$ of patients continued to have auras following surgery. Driving is of great importance to individuals with epilepsy and is a key benefit of seizure freedom. ${ }^{45}$ In the UK, the Driver and Vehicle Licencing Agency (DVLA) determines driving regulations. The most commonly applied rule is the need to be clear of seizures for 1 year to be eligible for a Group 1 licence (private motor car).

In USA, the required seizure-free interval varies from state to state with restrictions ranging from 3 months to 12 months. ${ }^{6}$ In Pennsylvania, an individual may drive providing a 'specific prolonged aura accompanied by sufficient warning has been established over a period of at least 2 years immediately preceding, with or without medication'? Some states, such as Indiana, have no set interval and an individual may drive so long as they are able to present a letter from a licenced physician stating they are taking effective medication. ${ }^{8}$

Currently in UK, patients with auras (simple partial seizures, focal seizures without impairment of awareness) are permitted to drive if this pattern is established over 12 months and if they have never had seizures which impaired awareness. ${ }^{9}$ In an epilepsy surgical population, it is highly unlikely for a patient to have never had a seizure with impaired consciousness. In consequence, postsurgical patients with continued auras do not qualify for a UK Group 1 driving licence.

\section{Calculating risk}

The risk of epilepsy causing a driving accident depends on several factors. The chance of an occurrence of a seizure within the next year (COSY) is the expected seizure rate in a next given year and can be expressed as $C$. $R$ denotes the relative risk of a patient having an accident in comparison to unaffected members of the population. At present, the DVLA allows individuals to have a Group 1 licence if they are perceived to have a risk of seizure in the next year (COSY) of $<20 \%$. The following formula demonstrates the relationship between the COSY, relative risk $(R)$ of a subject group compared with the overall driving population and other variables $^{10} 11$ :

$$
C=(R-1) \times(F / D X)
$$

$C=\operatorname{COSY}$

$R=$ relative risk.

$F=$ present fatal casualty rate per driver per year.

$D=$ time spent behind the wheel.

$X=$ probability of a seizure at the wheel leading to a serious injury or fatality.

The European Working Group on Epilepsy previously noted that individual risk, $R$, is increased by many commonplace characteristics such as driver age $>75$ years $(R=3.2)$ and male drivers $<25$ years 
$(R=7) .{ }^{11}$ These groups are permitted to drive and so it could be argued that other populations with $R \leq 3$ should also be allowed to drive.

Derivation of $R$ using the above formulae with UK statistics is as follows. For a private driver, the time spent behind the wheel $D$ is estimated at $4 \%$ of the average day or 1 hour. ${ }^{12}$ In Great Britain in 2014, there were 146322 personal injury accidents reported to the police and 194477 casualties (injuries of all severity). ${ }^{13}$ Of the 194477 casualties, 1775 people were killed. There are currently 38000000 drivers with a full license in the UK (https:// data.gov.uk/dataset/driving-licence-data). If 38000000 drivers cause 1775 fatalities per year, then the annual fatality rate, $F$, would be roughly 1 fatality per 23000 drivers (0.000044). X is the probability that a seizure while driving leads to a fatality. Approximately $60 \%$ of seizures behind the wheel lead to an accident. ${ }^{11} X$ is therefore the chance an accident leads to a fatality $(1775 / 146322)$ multiplied by 0.6 giving $X=0.0073$. It is possible that an accident caused by a seizure is more likely to lead to a fatality, but this is uncertain. ${ }^{11}{ }^{14}$ Putting these values into the equation gives $F / D X=0.15$. Therefore, using our original equation, if $C=0.2$ (a $20 \%$ chance of a seizure within the next year), then $R=2.33$. If $C=0.4$, then $R=3.67$.

Thus, for UK, a 20\% chance of a seizure within the next year would provide a relative risk, $R$, of 2.33 , a figure below the proposed $R=3$ and hence concordant with the DVLA policy of requiring a COSY below 20\% in order to drive.

At present, the occurrence of auras only following surgery prohibits driving, as nearly all individuals will have had prior seizures with loss of consciousness. Approximately $10 \%$ of patients who have undergone epilepsy surgery continue to have auras only. ${ }^{2}$ These patients are not currently allowed to drive in UK. The risk of allowing these patients to drive has not been quantified. This study aimed to estimate the risks of this population having a seizure with impairment of consciousness in the following year and hence whether this population should be permitted to drive.

\section{METHODS}

Data were collected from 950 operations on patients who had undergone epilepsy surgery at The National Hospital for Neurology and Neurosurgery between February 1990 and January 2014. Of these, 131 procedures were excluded (106 due to incomplete data or because surgery had taken place within the last 12 months and 25 as they were second operations in patients). Data were acquired from GP records, hospital notes and direct patient contact.

In total, 819 patients were included. The number of postoperative years of follow-up per patient range from 2 to 25 (mean $=11.4$ years, $S D=6.2$ years), with a total 9307 years of follow-up. Table 1 summarises characteristics of the patient cohort.

Data were anonymised with each patient receiving a unique patient identifier. Information recorded for each patient included operation date, operation type, laterality of surgery, surgeon and neuropathology. For each year of follow-up, the patient had been prospectively graded according to survival and the ILAE epilepsy surgery outcome scale (table 2) regarding occurrence of seizures in the previous 12 months. Patients were followed up until either death or loss to follow-up. There were 34 patients (4.2\%) who died during follow-up with various causes of death. Since the proportion of deaths in the cohort was small, mortality was not modelled explicitly. Data were analysed using Stata (V.14) for data summaries and R (V.3.1.3) for modelling.

\begin{tabular}{llc}
\hline Table 1 & Cohort characteristics & \\
\hline Variable & Category & Frequency \\
\hline Operation type & T Lob & $633(77.3 \%)$ \\
& T Les & $58(7.1 \%)$ \\
& Ex-T Lob & $58(7.1 \%)$ \\
\hline & Ex-T Les & $49(6.0 \%)$ \\
& MST & $3(0.3 \%)$ \\
\hline Side & Hemi & $14(1.7 \%)$ \\
& C Cal & $4(0.5 \%)$ \\
\hline \multirow{4}{*}{ Pathology } & Left & $427(52.0 \%)$ \\
& Right & $387(47.3 \%)$ \\
& N/A & $6(0.7 \%)$ \\
& HS & $498(60.8 \%)$ \\
& GL & $28(3.4 \%)$ \\
& CAV & $51(6.2 \%)$ \\
& DNT & $93(11.4 \%)$ \\
& FCD & $36(4.4 \%)$ \\
& Dual pathology & $16(2.0 \%)$ \\
& Other & $97(11.8 \%)$ \\
\hline
\end{tabular}

CAV, cavernoma; C Cal, corpus callosotomy; DNT, dysembryoplastic neuroepithelial tumour; Ex-T Les, extra temporal lesionectomy; Ex-T Lob, extra temporal lobectomy; FCD, focal cortical dysplasia; GL, glioma; Hemi, hemispherectomy; $\mathrm{HS}$, hippocampal sclerosis; MST, multiple subpial transection; T Lob, temporal lobectomy; T Les, temporal lesionectomy.

Principally, we considered ILAE grades 1 and 2, since any patient with ILAE grade $\geq 3$ would not usually be permitted to drive (unless seizures only occurred during sleep for more than 3 years). As such, ILAE grades $\geq 3$ are grouped together for the rest of this study.

First, basic tables of the seizure variable aggregated across all years of follow-up, conditional on the past history of ILAE grade were produced. Estimates of the COSY were obtained together with 95\% CIs using a linear regression model. The CIs did not account for the clustering of observations within patients.

To obtain marginal estimates of the probability of seizure occurrence, conditional on the past history of ILAE grade and accounting for the clustering of observations over time within patients, we used a generalised estimating equations (GEE) model with a binary outcome. Each GEE fit assumed an independence working correlation structure to avoid the possibility of bias in the model coefficient estimates. ${ }^{15}$ Robust SEs were used for the calculation of $95 \%$ CIs to account for within-patient clustering.

Each model has the form:

$$
\log \left(\frac{p_{i j}}{1-p_{i j}}\right)=\beta X_{i j}
$$

\section{Table 2 The ILAE epilepsy surgery outcome classification ${ }^{3}$}

\begin{tabular}{|c|c|}
\hline $\begin{array}{l}\text { Outcome } \\
\text { classification }\end{array}$ & Definition \\
\hline 1 & Seizure-free, no auras \\
\hline 2 & Simple partial seizures/auras only \\
\hline 3 & One to three seizure days per year+l- auras \\
\hline 4 & $\begin{array}{l}\text { Four seizure days per year to a } 50 \% \text { reduction in seizure days } \\
\text { per year+/-auras }\end{array}$ \\
\hline 5 & $\begin{array}{l}\text { Less than } 50 \% \text { reduction in seizure days to a } 100 \% \\
\text { increase }+/ \text {-auras }\end{array}$ \\
\hline 6 & A more than $100 \%$ increase in seizure days + -auras \\
\hline
\end{tabular}

ILAE, The International League against Epilepsy. 
Table 3 Risk of seizure with impaired awareness conditional on highest ILAE grade in previous 1, 2 and 3 years

\begin{tabular}{|c|c|c|c|}
\hline & \multicolumn{2}{|c|}{ Seizure in year } & \multirow{2}{*}{$\begin{array}{l}\text { Proportion of patients who } \\
\text { have a seizure }(95 \% \mathrm{Cl})\end{array}$} \\
\hline & Yes & No & \\
\hline \multicolumn{4}{|c|}{ Previous year's ILAE grade $(n=819)$} \\
\hline 1 & 237 & 4644 & 0.049 (0.041 to 0.056$)$ \\
\hline 2 & 101 & 790 & $0.113(0.096$ to 0.131$)$ \\
\hline$\geq 3$ & 2380 & 336 & $0.876(0.867$ to 0.886$)$ \\
\hline \multicolumn{4}{|c|}{ Highest ILAE grade in previous 2 years $(n=770)$} \\
\hline 1 & 143 & 3958 & 0.035 (0.026 to 0.044$)$ \\
\hline 2 & 74 & 734 & $0.092(0.072$ to 0.111$)$ \\
\hline$\geq 3$ & 2237 & 523 & 0.811 (0.800 to 0.821$)$ \\
\hline \multicolumn{4}{|c|}{ Highest ILAE grade in previous 3 years $(n=724)$} \\
\hline 1 & 84 & 3408 & 0.024 (0.014 to 0.034$)$ \\
\hline 2 & 56 & 662 & $0.077(0.056$ to 0.100$)$ \\
\hline$\geq 3$ & 2055 & 634 & 0.764 (0.753 to 0.776$)$ \\
\hline
\end{tabular}

ILAE, The International League against Epilepsy.

\section{With}

$p_{i j}=$ probability that patient $i$ undergoes a seizure in year $j$.

$\beta=$ group of model coefficients to be estimated.

$X_{i j}=$ group of patient-specific covariates measured at time point $j$.

\section{RESULTS}

Table 3 shows the proportion of patients who had a seizure with impairment of awareness in any given year, according to the previous year's ILAE grade. Thus, for postoperative patients whose previous year ILAE grade $=1$, the estimated probability of a seizure in the next year (the COSY) was 0.049 or $4.9 \%$. For those with ILAE outcome grades 1 and 2, there was progressively less risk of a seizure with loss of awareness, if the pattern was established for 2 or 3 years.

In view of within-patient clustering, the GEE approach was used to estimate further COSY values for each group. We present three basic models for the marginal estimates of the probability of undergoing a seizure with impaired awareness in the following year.

Table 4 also shows the risk of a seizure based on highest ILAE grade in the previous 2 and 3 years respectively. The results are

\begin{tabular}{ll}
\hline $\begin{array}{l}\text { Table } 4 \\
\text { year's ILAE grade }\end{array}$ & $\begin{array}{l}\text { Estimated proportion of patients who } \\
\text { undergo a seizure }(95 \% \mathrm{Cl})\end{array}$ \\
\hline & $0.049(0.041$ to 0.057$)$ \\
\hline Previous year's ILAE grade & $0.113(0.091$ to 0.140$)$ \\
\hline 1 & $0.876(0.856$ to 0.894$)$ \\
2 & $0.035(0.029$ to 0.042$)$ \\
$\geq 3$ & $0.092(0.072$ to 0.116$)$ \\
\hline Highest ILAE grade in previous 2 years & $0.811(0.782$ to 0.836$)$ \\
\hline 1 & $0.024(0.019$ to 0.030$)$ \\
2 & $0.078(0.060$ to 0.101$)$ \\
\hline 33 & $0.764(0.730$ to 0.795$)$ \\
\hline 1 &
\end{tabular}

ILAE, The International League against Epilepsy.
Table 5 Generalised estimating equation model with ILAE grade at previous year and years since surgery (year)

\begin{tabular}{ll}
\hline Explanatory variable & OR* \\
\hline ILAE grade in previous year $=2$ & $2.50(1.87$ to 3.35$)$ \\
ILAE grade in previous year $\geq 3$ & $138.77(106.41$ to 180.97$)$ \\
Year & 1.00 (0.99 to 1.02$)$ \\
\hline
\end{tabular}

${ }^{*} \mathrm{OR}$ in comparison to the category: previous ILAE grade $=1$.

ILAE, The International League against Epilepsy.

similar to those in table 3, but the $95 \%$ CIs are slightly different, when accounting for the clustering within patients over time.

These models estimate overall, marginal, estimates of probabilities conditional on past ILAE grade history, but do not account for important patient-level covariates or possible changes over time. We therefore fitted GEE models that include 'year' (time in years since surgery) as a covariate (table 5), to account for the possibility of a linear trend over time. For these models, we report ORs together with associated 95\% CIs.

The covariate 'year' did not influence the likelihood of a disabling seizure (OR 1.00; 0.99 to 1.02). However, scoring ILAE grade 2 in a previous year does (OR 2.50; 1.87 to 3.35 ) as does a grade 3 or more in the previous year (OR 138.77; 106.41 to 180.97$)$.

Current DVLA guidelines are predicated on the basis that patients who are seizure-free for 1 year have a COSY below $20 \%$. In our GEE model, patients who were completely seizurefree for 1 year had a $4.9 \%$ chance of a seizure with loss of awareness within the next 12 months. A COSY of $4.9 \%$ would give an individual risk value of $R=1.33$. Those with auras had a risk of $11.3 \%$ of such a seizure in the next year, giving an individual risk value of $R=1.75$ (table 6).

\section{DISCUSSION}

\section{Key findings}

The COSY for a patient with an ILAE grade 1 in any given year was estimated to be $4.9 \%$ with the upper bound of the $95 \%$ CI less than 20\%. This result supports current UK driving law, presuming the acceptable risk level is agreed on. Current UK driving law holds that a COSY below 20\% indicates an acceptable risk for driving. Patients with auras only in the previous 1 year had an estimated COSY of $11.3 \%$, with an upper $95 \%$ CI of $14 \%$. Risk was reduced if auras or simple partial seizures only had been present over the previous 2 or 3 years $(9.2 \%$ and $7.8 \%$ respectively).

Patients with a seizure with loss of awareness in the previous year had an $87.6 \%$ risk of such a seizure in the next year, with a consequent relative risk of an accident of 6.84. It is notoriously difficult to be certain about the numbers of auras experienced by a patient as these usually occur at irregular intervals and frequency is not accurately recorded by individuals, so we cannot determine whether the frequency of recalled auras is a factor in predicting risk of a seizure with impairment of awareness. In clinical practice, on which decisions about driving are made, neurologists rely on the history provided by patients and

Table 6 Individual risk based on previous year's ILAE grade

\begin{tabular}{ll}
\hline Previous year's ILAE grade & Relative risk, $\boldsymbol{R}$ \\
\hline 1 & 1.33 \\
2 & 1.75 \\
$\geq 3$ & 6.84 \\
\hline ILAE, The International League against Epilepsy.
\end{tabular}


witnesses to decide whether there is any impairment of awareness associated with reported brief focal seizures or auras. In general, auras do not occur at a frequency that would make video-EEG telemetry recordings a practical proposition.

If individual risk is considered, patients with grade 1 or grade 2 outcomes in any given year have a less than threefold risk of an accident ( $R=1.33$ and 1.75 respectively). These grades imply less of an increase in relative risk than increases associated with other characteristics that do not prevent driving such as age over 75 , females $<25$ years and males $<25$ years $(R=3.1,3.2$ and 7 respectively).

\section{Implications for driving law}

Although patients with only auras have a raised COSY, the increase is relatively small and these patients pose a similar risk to those who are entirely seizure-free. The European Working Group suggested that a COSY between 20\% and 40\% may be acceptable given the risks that drivers with other circumstances, both medical and biographical, may pose.

When other factors, such as the probability of a seizure occurring at the wheel and of a seizure with loss of awareness causing an accident are considered, the overall increase in relative risk is small at 1.75 for the aura group and relatively low in comparison to other groups of drivers such as those aged $<25$ or $>75$ years. This risk estimate also relies on a number of assumptions such as driving for an average of 1 hour per day. The risk of a road traffic accident, and consequent injury, increases with the time spent driving. This is one of the reasons for the criterion for a Group 2 licence being a $<2 \%$ annual risk of a seizure.

Given the significant potential improvements in quality of life and the relatively low individual risk increase in comparison to other groups, an argument could be made that patients with auras only following epilepsy surgery could be considered safe enough to possess a Group 1 driving licence.

Limited licences have been considered for epilepsy and are in place for other medical conditions. ${ }^{11}$ Measures that could be considered include limiting time behind the wheel, banning the carriage of passengers, the avoidance of motorways and a $0 \%$ alcohol limit. These suggestions, however, would be difficult to police and may compromise the improvement in quality of life that patients expect.

Many medical conditions such as diabetes and heart disease increase the risk of an accident. ${ }^{6}$ Problems may arise when, as is often the case, patients have multiple comorbidities that individually may not exclude them from driving but together pose a potentially dangerous mix. Waller et $a l^{16}$ suggested that cardiovascular disease carries a 1.62 relative risk of an accident, diabetes 1.78 and substance abuse 2.8. The risk for patients with cardiovascular disease and diabetes, a common combination, is 2.88 , higher than that of the aura only group in the current study.

In conclusion, using a simple GEE, we calculated the chance of a seizure with loss of awareness in the next year for multiple patient groups on the ILAE epilepsy surgery scale. Individuals having auras only after epilepsy surgery have a COSY of below the suggested $20 \%$ and a relative risk of 1.75 . Given the improvements in quality of life that are possible through the liberalisation of driving law and the potential to reduce risk through limited licencing, it may be reasonable to suggest that patients with auras only following epilepsy surgery be allowed to drive.

Acknowledgements We are grateful for the support of the UK Department of Health National Institute for Health Research (NIHR) Biomedical Research Centres funding scheme and to Catrin Tudur-Smith, Liverpool University, for comments and independent statistical review of the final draft.

Contributors JSD conceived this evaluation and did data interpretation and editing. JdT collected the data and maintained the database. SF did the data analysis and drafted the paper. AGOK provided statistical support and data interpretation. All approved the final version.

Competing interests None declared.

Provenance and peer review Not commissioned; externally peer reviewed.

(c) Article author(s) (or their employer(s) unless otherwise stated in the text of the article) 2018. All rights reserved. No commercial use is permitted unless otherwise expressly granted.

\section{REFERENCES}

1 Wiebe S, Blume WT, Girvin JP, et al. A randomized, controlled trial of surgery for temporal-lobe epilepsy. N Eng/ J Med 2001;345:311-8.

2 de Tisi J, Bell GS, Peacock JL, et al. The long-term outcome of adult epilepsy surgery, patterns of seizure remission, and relapse: a cohort study. Lancet 2011;378:1388-95

3 Wieser HG, Blume WT, Fish D, et al. ILAE Commission report. Proposal for a new classification of outcome with respect to epileptic seizures following epilepsy surgery. Epilepsia 2001;42:282-6.

4 Bower CM, Hays RD, Devinsky 0, et al. Expectations prior to epilepsy surgery: an exploratory comparison of men and women. Seizure 2009;18:228-31.

5 Elsharkawy AE, May T, Thorbecke R, et al. Long-term outcome and determinants of quality of life after temporal lobe epilepsy surgery in adults. Epilepsy Res 2009:86:191-9.

6 Fisher RS, Parsonage M, Beaussart M, et al. Epilepsy and driving: an international perspective. joint commission on drivers' licensing of the international bureau for epilepsy and the international league against epilepsy. Epilepsia 1994;35:675-84.

7 Department of Transport Pennsylvania. Physical and mental criteria including vision standards relating to the licensing of drivers. Department of Transport Pennsylvania, 2010.

8 Epilepsy Foundation. State driving laws database 2014. 2016. http://www.epilepsy. com/driving-laws/2008711

9 Driver and Vehicle Licensing Agency. DVLA's current medical guidelines for professionals. UK: Driver and Vehicle Licensing Agency, 2015.

10 Spencer MB. The role of risk analysis in the evaluation of fitness to drive. UK: Department for Transport, 2003.

11 Second European Working Group on Epilepsy and Driving. Epilepsy and driving in Europe. Europe: Second European Working Group on Epilepsy and Driving, 2005.

12 Tranter MR, Goodman G D. National travel survey: England 2014. England: Department for Transport, Office for National Statistics, 2015.

13 Lloyd D. Reported road casualties in Great Britain. England: Department for Transport, Office for National Statistics, 2015.

14 Taylor J, Chadwick D, Johnson T. Risk of accidents in drivers with epilepsy. J Neurol Neurosurg Psychiatry 1996;60:621-7.

15 Schildcrout JS, Heagerty PJ. Regression analysis of longitudinal binary data with timedependent environmental covariates: bias and efficiency. Biostatistics 2005;6:633-52.

R16 Waller JA. Chronic medical conditions and traffic safety: review of the California experience. N Engl J Med 1965;273:1413-20. 